\title{
Langerhans cells utilize CD1a and langerin to efficiently present nonpeptide antigens to T cells
}

\author{
Robert E. Hunger, ${ }^{1}$ Peter A. Sieling, ${ }^{1}$ Maria Teresa Ochoa, ${ }^{1}$ Makoto Sugaya, ${ }^{2}$ Anne E. Burdick, ${ }^{3}$ \\ Thomas H. Rea, ${ }^{4}$ Patrick J. Brennan, ${ }^{5}$ John T. Belisle, ${ }^{5}$ Andrew Blauvelt, ${ }^{2}$ Steven A. Porcelli, ${ }^{6}$ \\ and Robert L. Modlin'1,7,8
}

\begin{abstract}
1Division of Dermatology, Department of Medicine, David Geffen School of Medicine at the University of California, Los Angeles (UCLA), Los Angeles, California, USA. ${ }^{2}$ Dermatology Branch, Center for Cancer Research, National Cancer Institute, Bethesda, Maryland, USA. ${ }^{3}$ Department of Dermatology and Cutaneous Surgery, University of Miami, Miami, Florida, USA. ${ }^{4}$ Section of Dermatology, Keck School of Medicine at the University of Southern California, Los Angeles, Los Angeles, California, USA. ${ }^{5}$ Mycobacterial Research Laboratories, Department of Microbiology, Immunology, and Pathology, Colorado State University, Fort Collins, Colorado, USA. ${ }^{6}$ Department of Microbiology and Immunology, Albert Einstein College of Medicine, Bronx, New York, USA.

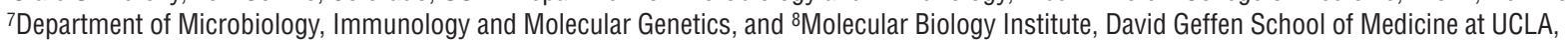
Los Angeles, California, USA.

Langerhans cells (LCs) constitute a subset of DCs that initiate immune responses in skin. Using leprosy as a model, we investigated whether expression of CD1a and langerin, an LC-specific C-type lectin, imparts a specific functional role to LCs. LC-like DCs and freshly isolated epidermal LCs presented nonpeptide antigens of Mycobacterium leprae to $\mathrm{T}$ cell clones derived from a leprosy patient in a CD1a-restricted and langerin-dependent manner. LC-like DCs were more efficient at CD1a-restricted antigen presentation than monocyte-derived DCs. LCs in leprosy lesions coexpress CD1a and langerin, placing LCs in position to efficiently present a subset of antigens to $T$ cells as part of the host response to human infectious disease.
\end{abstract}

\section{Introduction}

Dendritic cells (DCs) link the innate to the adaptive immune response by their ability to detect and capture foreign antigens and efficiently present these antigens to $T$ cells. Although all DCs share this capacity to present antigens to $\mathrm{T}$ cells, different DC subsets have been characterized according to their tissue distribution, cell surface markers, and their ability to instruct different patterns of adaptive immune responses.

Paul Langerhans, as a medical student, identified DCs resident in the skin, specifically the epidermis, that could be labeled with gold. So-called Langerhans cells (LCs) have been shown to be the only cells in the epidermis to express MHC class II molecules under normal conditions $(1,2)$. Upon antigen capture, LCs migrate to regional lymph nodes and are highly efficient at presenting MHC class II-restricted peptides to $\mathrm{T}$ cells $(3,4)$. These $\mathrm{T}$ cells then migrate back to the skin to participate in cutaneous inflammatory responses. These observations suggest that LCs are an important component of the innate immune response in skin, functioning to initiate adaptive $\mathrm{T}$ cell responses locally.

Two other features of LCs are intriguing in the context of their antigen-presenting role. First, LCs were shown to express high levels of $\mathrm{CD} 1 \mathrm{a}(5)$, a member of the family of group $1 \mathrm{CD} 1$ proteins (CD1a, CD1b, and CD1c), which share the capacity to present microbial lipid antigens to T cells (6-9). Second, LCs are the only cells known to express langerin (CD207) (10), a C-type lectin that is sufficient to induce the formation of Birbeck granules, pentilaminar endosomal structures specific to LCs (11). These unique fea-

Nonstandard abbreviations used: Langerhans cell (LC); lipoarabinomannan (LAM); median fluorescence intensity (MFI); monocyte-derived DC (MD-DC); mycolyl arabinogalactin peptidoglycan (MAGP); T cell receptor (TCR).

Conflict of interest: The authors have declared that no conflict of interest exists. Citation for this article: J. Clin. Invest. 113:701-708 (2004). doi:10.1172/JCI200419655. tures of LCs suggested that they may be specialized by their expression of langerin to capture particular antigens as they enter the epidermal layers, and that at least some of these antigens may be presented to CD1a-restricted T cells. In the current study, we have examined this hypothesis in the context of leprosy, a skin disease caused by infection with the obligate intracellular pathogen Mycobacterium leprae. The skin lesions of leprosy are readily accessible to study, and biopsy specimens are often taken to confirm the diagnosis. We have successfully used such specimens for immunologic studies and to derive $\mathrm{T}$ cell clones for in vitro analysis. Our results provide evidence that LCs are indeed highly efficient at presenting nonpeptide mycobacterial antigens to CD1a-restricted $T$ cells, and they implicate langerin as an important antigen-uptake receptor for the generation of such $\mathrm{T}$ cell responses.

\section{Methods}

Patients and clinical specimens. Leprosy patients were recruited on a volunteer basis from the ambulatory population seen at the Dermatology Clinics at the Medical Centers of the University of Southern California and the University of Miami with institutional review board approval. Clinical classification of patients with symptomatic M. leprae infection was done according to the criteria of Ridley and Jopling (12). Skin-biopsy specimens (6 $\mathrm{mm}$ in diameter) containing both epidermis and dermis were obtained by standard punch technique following informed consent. Blood samples for isolation of PBMCs were obtained by venipuncture from leprosy patients and from healthy volunteer laboratory personnel who served as a source of monocyte-derived DCs (MD-DCs). PBMCs were isolated using Ficoll-Hypaque gradient centrifugation (Ficoll-Paque; Pharmacia Biotech AB, Uppsala, Sweden). Samples of human cord blood were obtained from Santa Monica-UCLA Medical Center (Santa Monica, California, USA) and Saint John's Health Center (Santa Monica, California, USA). CD34 $4^{+}$human progenitor cells were isolated using 
RosetteSep (StemCell Technologies, Vancouver, British Columbia, Canada) according to the supplier's instructions.

Antigens and antibodies. Extracts of M. leprae, Mycobacterium tuberculosis, and other mycobacteria were prepared by probe sonication as previously described (13). Cellular subfractions and purified CD1 lipid antigens from mycobacteria were prepared as previously described (6-9). To enrich for nonpeptide antigens, extracts were in some cases treated with proteinase $\mathrm{K}$ as previously described (14). The following antibodies were used for CD1 neutralization studies, flow cytometry, and immunohistochemistry studies: OKT6 (anti-CD1a; ref. 15; neutralization), NA1/34 (anti-CD1a; DakoCytomation California Inc., Carpinteria, California, USA; immunohistochemistry), HI149 (anti-CD1a; Pharmingen, San Diego, California, USA; flow cytometry), BCD1b3.1 (anti-CD1b; ref. 16), F10/21A3 (anti-CD1c; ref. 17), S4.1 (anti-CD3; Caltag Laboratories Inc., Burlingame, California, USA), S3.5 (anti-CD4; Caltag Laboratories Inc.), 3B5 (anti-CD8 $\alpha$; Caltag Laboratories Inc.), BU15 (anti-CD11c; Caltag Laboratories Inc.), M5E2 (anti-CD14; Pharmingen), 1C3 (anti-CD58; Pharmingen), TÜ36 (HLA-DR; Caltag Laboratories), L307.4 (anti-CD80; Pharmingen), HB15e (antiCD83; Pharmingen), DCGM4 (anti-langerin; Beckman-Coulter Inc., Miami, Florida, USA), DCN46 (anti-DC-SIGN; Pharmingen), G46-6 (anti-HLA-DR; Pharmingen), BMA 031 (anti-T cell receptor $\alpha \beta$ [anti-TCR $\alpha \beta$ ]; Caltag Laboratories Inc.), 5A6.E9 (anti$\mathrm{TCR} \gamma \delta$; Caltag Laboratories Inc.), and appropriate isotype controls (Caltag Laboratories Inc. and Pharmingen).

In vitro culture of CD1-expressing LC-like DCs and MD-DCs. CD1expressing MD-DCs were induced in vitro with a combination of recombinant human GM-CSF $(200 \mathrm{U} / \mathrm{ml})$ and recombinant human IL-4 (100 U/ml) (PeproTech Inc., Rocky Hill, New Jersey, USA) for 72 hours as previously described $(6,18)$. Cells were incubated in PBS/0.5 mM EDTA to detach adherent cells and analyzed by flow cytometry to determine surface expression of CD1 or irradiated (50 Gy) and used as APCs as previously described (6). LC-like DCs derived from human cord blood were prepared as previously described (11). Briefly, cultures of $\mathrm{CD} 34^{+}$cells were established in the presence of stem cell factor $(25 \mathrm{ng} / \mathrm{ml}$; R\&D Systems Inc., Minneapolis, Minnesota, USA), GM-CSF (200 U/ml; Berlex Inc., Montville, New York, USA), and TNF- $\alpha(2.5 \mathrm{ng} / \mathrm{ml}$; Biosource International, Camarillo, California, USA). At day 8, cultures were replated in the presence of GM-CSF (200 U/ml; Berlex Inc.) and TGF- $\beta_{1}(1$ $\mathrm{ng} / \mathrm{ml}$; R\&D Systems Inc.) to increase CD1a expression. LC-like DCs were harvested at day 12-14.

Preparation of human epidermal LCs. The Institutional Review Board of the National Cancer Institute approved acquisition of human skin, and informed consent was obtained from all healthy volunteers. Blisters were induced by vacuum suction and heat on normalappearing skin. Blister roofs (i.e., epidermal sheets) were then removed with sterile scissors, washed three times in sterile PBS, placed in $0.5 \%$ trypsin/PBS for 45 minutes, and then mechanically disrupted to create single-cell suspensions. For some experiments, LCs were depleted from epidermal cell suspensions using anti-HLADR mAb's (Pharmingen) and immunomagnetic bead separation as described previously (19). Efficiency of LC depletion was confirmed by staining of depleted and undepleted cell populations with antiCD1 a mAb (HI149; Pharmingen), which specifically labels LCs within human epidermal cell suspensions. The percentage of $\mathrm{CD} 1 \mathrm{a}^{+}$cells in freshly prepared epidermal cell suspensions was $3.91 \% \pm 1.07 \%$, and that in LC-depleted populations was $0.01 \% \pm 0.01 \%$ (from three independent experiments).
T cell lines and proliferation assays. T cell lines were derived from blood of leprosy patients as previously described $(7,20)$. CD4-, CD8- double negative $\mathrm{T}$ cells, isolated from peripheral blood using RosetteSep CD4 enrichment and CD4 depletion cocktail simultaneously (StemCell Technologies), were cocultured with $M$. leprae in the presence of cord blood-derived LC-like DCs prepared as described above. T cell lines were maintained by serial antigenic stimulation in medium supplemented with recombinant IL-2 (1 nM; Chiron Diagnostics, Norwood, Massachusetts, USA). Heterologous irradiated PBMCs and phytohemagglutinin (Sigma-Aldrich, St. Louis, Missouri, USA) were used to further propagate $\mathrm{T}$ cell lines and to generate clones using limiting dilution (20). For measurement of antigen-specific proliferation, T cells $\left(1 \times 10^{4}\right)$ were cultured with varying numbers (usually $1 \times 10^{4}$ ) of irradiated (50 Gy) LC-like DCs or MD-DCs in 0.2 $\mathrm{ml}$ culture medium in the presence or absence of $5 \mu \mathrm{g} / \mathrm{ml}$ bacterial antigens for 3 days in microtiter wells in triplicate at $37^{\circ} \mathrm{C}$ in a $7 \%$ $\mathrm{CO}_{2}$ incubator. Cells were pulsed with ${ }^{3} \mathrm{H}$-thymidine $(1 \mu \mathrm{Ci} /$ well; ICN Biomedicals Inc., Costa Mesa, California, USA) and harvested 4-6 hours later for liquid scintillation counting. To determine CD1restriction of the $\mathrm{T}$ cell lines, neutralizing CD1 antibodies $(20 \mu \mathrm{g} / \mathrm{ml})$ were added 30 minutes before the addition of $\mathrm{T}$ cells. To determine the role of langerin (CD207), neutralizing antibodies to langerin (20 $\mu \mathrm{g} / \mathrm{ml}$ ) were added to the culture 30 minutes before the addition of antigen and T cells. Supernatants were removed from the T cell cultures at 24 hours, and IFN- $\gamma$ was measured by ELISA (IFN- $\gamma$; Pharmingen) according to the manufacturer's instructions.

Immunohistochemical studies. Biopsy specimens were embedded in OCT medium (Sakura Finetek USA Inc., Torrance, California, USA) and snap-frozen in liquid nitrogen. Sections (3-5 $\mu \mathrm{m}$ thick) were acetone-fixed and blocked with normal horse serum and then incubated with the mAb's for 60 minutes, followed by biotinylated horse anti-mouse IgG for 30 minutes. Primary antibody was visualized with the Vectastain Elite ABC system (Vector Laboratories Inc., Burlingame, California, USA), which uses avidin and a biotin-peroxidase conjugate for signal amplification. ABC reagent was incubated for 30 minutes, washed, then incubated with substrate (3-amino-9-ethylcarbazole) for 10 minutes. Slides were counterstained with hematoxylin and mounted in crystal mounting medium (Biomeda Corp., Foster City, California, USA).

Two-color immunofluorescence staining of cryostat sections. Double immunofluorescence was performed by serial incubation of cryostat tissue sections with mouse anti-human mAb's of different isotypes (e.g., NA1/34 [anti-CD1a, IgG2a] and anti-langerin [anti-CD207, IgG1]), followed by incubation with isotype-specific goat anti-mouse Ig antibodies (Molecular Probes Inc., Eugene, Oregon, USA) labeled with fluorochrome (Alexa 488 or Alexa 568). Controls included staining with isotype-matched irrelevant antibodies, as well as staining with anti-CD1a or anti-langerin followed by secondary antibodies mismatched to the primary antibody isotype to demonstrate the isotype specificity of the secondary labeled antibodies. Images were obtained using confocal laser microscopy.

Confocal microscopy. Immunofluorescence was examined with a Leica TCS SP inverted confocal laser scanning microscope (Leica Microsystems Inc., Bannockburn, Illinois, USA) fitted with krypton and argon lasers at the Carol Moss Spivak Cell Imaging Facility in the UCLA Brain Research Institute. Sections and cells were illuminated with 488 and $568 \mathrm{~nm}$ of light after filtering through an acoustic optical device. Images decorated with Alexa 488 or Alexa 568 (Molecular Probes Inc.) were recorded simultaneously through separate optical detectors with a 530-nm band-pass filter and a 590-nm 
A

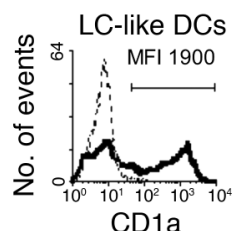

B
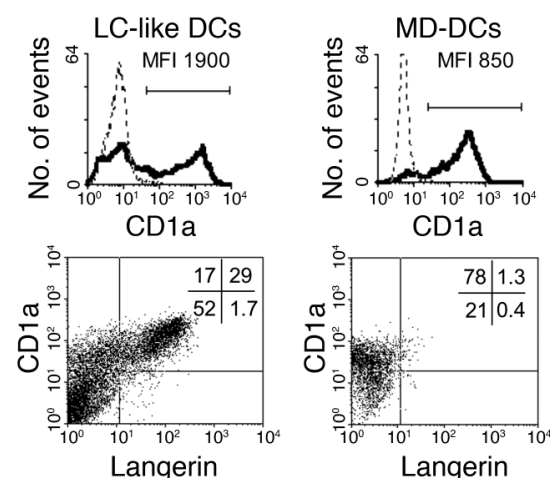

CD1a

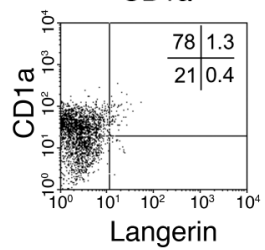

C

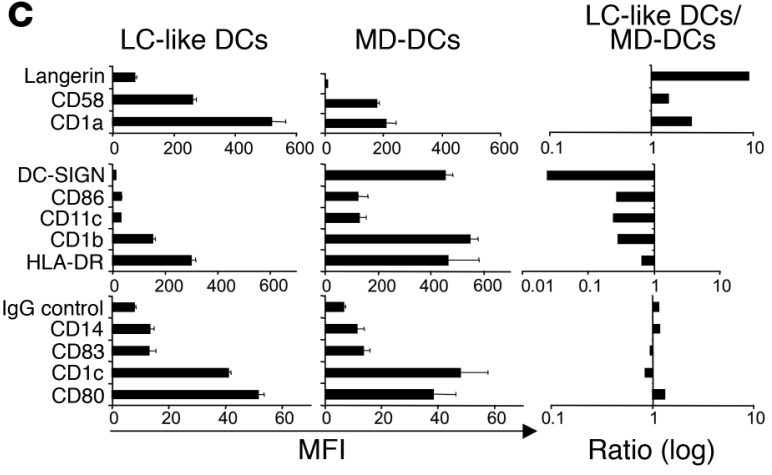

long-pass filter, respectively. Pairs of images were superimposed for colocalization analysis.

Statistical analysis. Statistical comparisons in $\mathrm{T}$ cell proliferation assays were made using Student's $t$ test. $P$ values of less than 0.05 were considered significant.

\section{Results}

Phenotype of in vitro-derived human LC-like DCs. Previous studies of presentation by group $1 \mathrm{CD} 1$ molecules (CD1a, CD1b, and CD1c) have used MD-DCs to activate T cells $(6,7,21)$. Given that MD-DCs express high levels of $\mathrm{CD} 1 \mathrm{~b}$, but variable levels of $\mathrm{CD} 1 \mathrm{a}$ and $\mathrm{CD} 1 \mathrm{c}$, it is not surprising that most of the $\mathrm{T}$ cell clones that have been established using these APCs were CD1b-restricted. In fact, to our knowledge, there is only one report of a single microbial reactive CD1a-restricted T cell clone (22). We reasoned that these DCs may not be optimal for presenting antigen via CD1a. Given that LCs are known to express CD1a, we derived LC-like DCs from fetal cord blood and compared their expression profile with that of MD-DCs. LC-like DCs and MD-DCs both expressed CD1a, although their patterns of expression were distinct. LC-like DCs expressed higher levels of CD1a than MD-DCs (median fluorescence intensity [MFI] 1,900 for LC-like DCs, 850 for MD-DCs), although the percentage of CD1a-expressing cells was greater in the MD-DCs (46\% of LC-like DCs positive, 79\% of MD-DCs positive) (Figure 1A). Expression of langerin is characteristic of LCs (10); therefore, we evaluated the expression of langerin on the two in vitro-derived DC populations. Langerin was present on $63 \%$ of the CD1a ${ }^{+}$LC-like DCs, but only $1.6 \%$ of the $\mathrm{CD} 1 \mathrm{a}^{+} \mathrm{MD}-\mathrm{DCs}$ (Figure $1 \mathrm{~B}$ ).

We next compared the expression of proteins involved in antigen presentation on the two DC populations. Figure 1C shows that langerin, CD58, and CD1a were expressed at higher levels on LC-like DCs than on MD-DCs. In contrast, DC-SIGN, CD86, CD11c, CD1b, and HLA-DR were expressed at higher levels on MD-DCs than on

\section{Figure 1}

Phenotype of cord blood-derived LC-like DCs and MD-DCs. (A) LC-like DCs and MD-DCs were stained with mAb's specific for CD1a (solid line) and mouse IgG control antibody (dashed line). MFI is indicated for the positive cell population. (B) LC-like DCs and MD-DCs were doublestained with mAb's specific for CD1a and langerin. The numbers indicate the percentage of cells in each quadrant. One representative experiment is shown from four independent donors. (C) LC-like DCs and MD-DCs were stained with numerous surface proteins. MFI was determined by flow cytometry. For each data point, cells from three different donors were analyzed ( \pm SEM). The ratios of MFI from LC-like DCs to MFI from MD-DCs were calculated and shown on a log scale as indicated.

LC-like DCs. Additional protein markers, CD14, CD83, CD1c and CD80, were equally expressed on the two DC populations (Figure $1 \mathrm{C}$, lower panel). These data indicate that LCs represent a unique subset of DCs, coexpressing both CD1a and langerin.

CD1a-restricted $T$ cell clones derived from leprosy patients. To evaluate the relative effectiveness of LCs for antigen presentation through CD1a, we derived CD1a-restricted $\mathrm{T}$ cell clones. $\mathrm{T}$ cell clones were derived using CD1 $\mathrm{a}^{+}$LC-like DCs and CD4- and CD8-depleted T cells from the blood of leprosy patients. In a first attempt to study CD1a-restricted $\mathrm{T}$ cells, we limited the experiments to the double negative T cell population, because allogeneic LC-like DCs were used as APCs. Nineteen clones were derived in this manner, of which 14 proliferated in the presence of extracts from $M$. leprae. These T cell clones expressed CD3 and TCR $\alpha \beta$, but no detectable CD4 or CD8 (Figure 2A). The proliferative response of seven of the $14 \mathrm{M}$. leprae-reactive clones was inhibited to some extent by anti-CD1a antibodies. Two $\mathrm{T}$ cell clones were selected for further study based on two criteria: (a) the ability to be maintained in culture; and (b) the extent to which anti-CD1 antibodies neutralized the $\mathrm{T}$ cell response

A
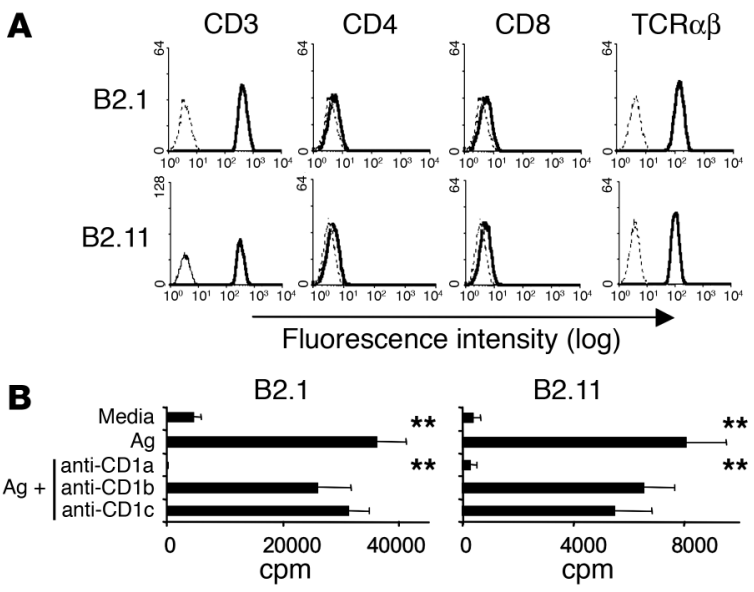

B2.11

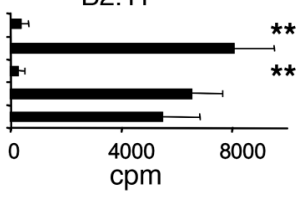

Figure 2

Phenotype and restriction pattern of antigen-specific T cell clones. (A) $\mathrm{T}$ cell lines B2.1 and B2.11 were analyzed for surface expression of CD3, CD4, CD8, and TCR $\alpha \beta$. (B) Cells were cultured with irradiated LC-like DCs in the presence or absence of antigen (Ag; sonicated $M$. leprae) with or without blocking antibodies as indicated. T cells were pulsed with ${ }^{3} \mathrm{H}$-thymidine after 72 hours of culture. Cells were harvested, and ${ }^{3} \mathrm{H}$ incorporation was measured by a scintillation counter. The values shown are the mean \pm SEM of triplicate cultures and are representative of at least three independent experiments. Student's $t$ test was used to compare cpm values of $T$ cells stimulated with antigen alone versus antigen plus neutralizing antibodies. ${ }^{* \star} P<0.005$. 

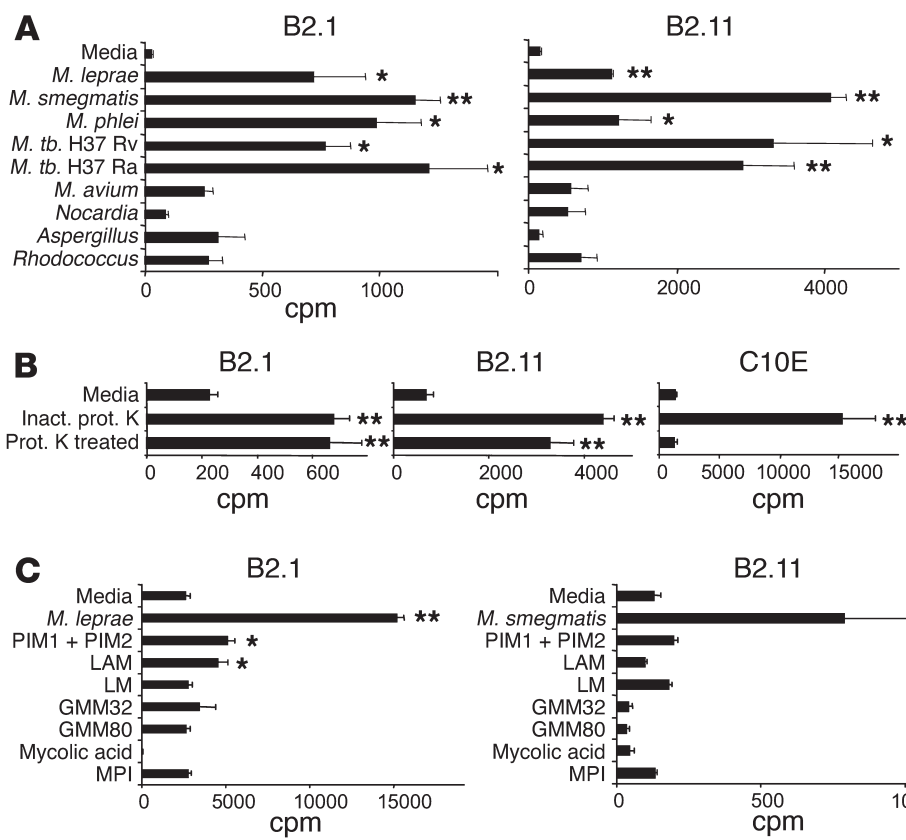

B2.11

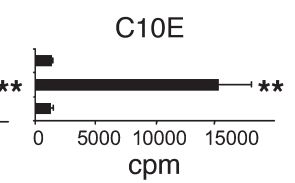

D
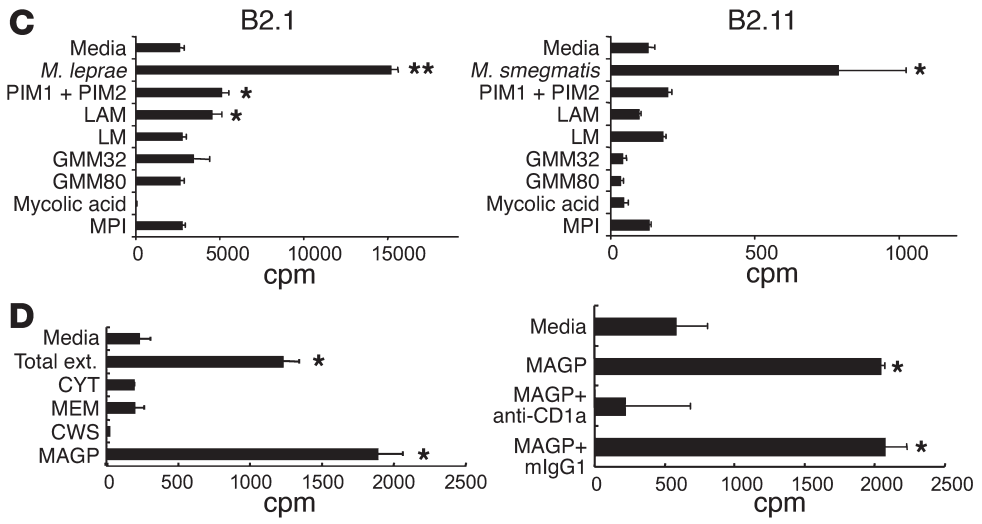

\section{Figure 3}

Specificity of the proliferative responses of CD1a-restricted T cell clones to different bacterial lysates and known CD1-restricted antigens. (A) T cell lines were tested for their ability to recognize different total bacterial sonicates. M. tb., M. tuberculosis. (B) M. leprae sonicate preparation was digested with proteinase $\mathrm{K}$. The proliferative response was determined using CD1a-restricted $T$ cell line B2.1, B2.11 and the MHC class II-restricted cell line $\mathrm{C} 10 \mathrm{E}$. Inact. prot. $\mathrm{K}$ indicates that the enzyme was heat-inactivated prior to adding to bacterial extract. (C) Proliferative response was measured using different known CD1b- and CD1c-restricted antigens. Proliferative responses were measured as described for Figure 2 using LC-like DCs as APCs for $\mathrm{T}$ cell clones B2.1 and B2.11 and MHC-matched PBMCs for the C10E T cell line. PIM, phosphatidyl-myo-inositol mannoside; LAM, lipoarabinomannan; LM, lipomannan; GMM, glucose monomycolate; MPI, mannosyl phosphoisoprenoid. (D) Characterization of mycobacterial antigen for CD1a-restricted T cells. Left: CD1a-restricted T cell clone B2.11 was cultured with three mycobacterial extracts containing cytosol (CYT), membrane (MEM), soluble cell wall material (CWS), and mycolyl arabinogalactin peptidoglycan (MAGP). Total ext., mycobacterial extract prior to fractionation. Right: T cell-responsive MAGP was examined for CD1a-restriction using neutralizing antibodies to CD1a. The values shown are the mean \pm SEM of triplicate cultures and are representative of at least three independent experiments. Statistical analysis was performed as indicated in the legend to Figure 2, but comparing $T$ cells stimulated with media versus antigen. ${ }^{\star} P<0.05 ;{ }^{\star \star} P<0.005$. to M. leprae. In five of the other seven $\mathrm{T}$ cell lines that responded to $M$. leprae, either $\mathrm{T}$ cell lines could not be maintained in vitro or antibodies to CD1a did not substantially prevent the response. By contrast, proliferation of $\mathrm{T}$ cell clones B2.1 and B2.11 in response to $M$. leprae was completely inhibited by neutralizing anti-CD1a mAb's (Figure 2B), whereas no inhibition was detected in the presence of other anti-CD1 antibodies, indicating that the two clones were restricted by CD1a. T cell clones B2.1 and B2.11 were therefore selected for analysis of antigen presentation through CD1a by LCs.

Antigen recognition by CD1a-restricted $T$ cell clones. CD1-restricted $\mathrm{T}$ cell clones recognize lipid antigens conserved among different species of mycobacteria. We evaluated the response of the two CD1arestricted $\mathrm{T}$ cell clones in the presence of various bacterial extracts to determine the antigen specificity. In addition to M. leprae, the two clones responded vigorously to several mycobacterial extracts, including M. tuberculosis, Mycobacterium smegmatis, and Mycobacterium phlei, but only weakly to Mycobacterium avium, Nocardia, Aspergillus, and Rhodococcus species (Figure 3A). The data indicate that the antigen recognized by the CD1a-restricted $\mathrm{T}$ cell clones is shared among number of bacteria with complex lipid envelopes.

To determine whether the antigen recognized by the CD1arestricted $\mathrm{T}$ cell clones was peptide or nonpeptide in nature, mycobacterial extracts were subjected to proteolysis. Proteolysis had no appreciable effect on antigen recognition by the CD1a-restricted $\mathrm{T}$ cell clones (Figure 3B) but completely abrogated the antigen recognition by the MHC class II-restricted T cell clone C10E (23), indicating that the antigen recognized by the CD1a-restricted clones is a nonpeptide, presumably a lipid. To determine whether the antigen or antigens presented by CD1a to the $\mathrm{T}$ cell clones were the same as or distinct from those presented by other group $1 \mathrm{CD} 1$ proteins, we tested the response of the CD1a-restricted $\mathrm{T}$ cell clones with a panel of known CD1 lipid antigens. The CD1a-restricted T cell clones responded only weakly to phosphatidyl-myo-inositol mannoside (7) and lipoarabinomannan (7), and not at all to glucose monomycolate (8), mycolic acid (24), or mannosyl phosphoisoprenoid (9) (Figure $3 \mathrm{C}$ ), indicating that the antigen presented by LC-like DCs is distinct from those presently characterized for other group 1 CD1 proteins.

To identify the nature of the nonpeptide antigen for CD1a-restricted T cells, we used crude extracts of mycobacteria encompassing the entirety of the mycobacterial cell (7). The four fractions were enriched for cytosolic, membrane, and cell wall components. The cell wall components were further differentiated into soluble and insoluble fractions. The fractions were tested for their ability to stimulate $\mathrm{T}$ cell clone B2.11. Mycolyl arabinogalactan peptidoglycan (MAGP), a fraction containing insoluble mycolic acids, arabinogalactan, and peptidoglycan, stimulated the $\mathrm{T}$ cell clone, while other fractions did not stimulate the T cells (Figure 3D). The response to MAGP was inhibited by neutralizing antibodies to CD1a (Figure 3D), confirming that the CD1a antigen is present in the MAGP fraction.

LC-like DCs are more efficient APCs for CD1a-restricted $T$ cells than $M D-D C$ s are. We compared the relative efficiency of LC-like DCs and MD-DCs for CD1a and CD1b antigen presentation. The numbers of APCs were normalized according to the frequency of CD1aexpressing cells. LC-like DCs, but not MD-DCs, stimulated the proliferation of CD1a-restricted T cell clones in response to $M$. leprae extracts (Figure 4A, left). In contrast, MD-DCs were more effective APCs for CD1b-restricted T cells (Figure 4A, right). Similarly, cytokine production from CD1a-restricted $\mathrm{T}$ cells was more robust when antigen was presented by LC-like DCs than when it was presented by MD-DCs (Figure 4B). These data indicated that LC-like 
DCs are more efficient than MD-DCs in presentation of antigen via CD1a to T cells. Although the obvious explanation was the greater expression of CD1a by LC-like DCs than by MD-DCs, we sought to determine whether langerin might also have a role in CD1a-restricted antigen presentation.

Langerin mediates the uptake and/or processing of antigen to CD1arestricted $T$ cells from in vitro-derived LC-like DCs and freshly isolated LCs from buman skin. Langerin is a C-type lectin expressed exclusively on LCs and plays a vital role in the formation of Birbeck granules (11), a characteristic intracellular organelle of LCs that is thought to represent a recycling/early-endosomal structure (25). Thus we wished to determine the role of langerin in CD1a antigen presentation. Addition of neutralizing anti-langerin mAb's at the initiation of culture efficiently blocked the proliferative response of both CD1arestricted $\mathrm{T}$ cell clones, but not the response of a CD1b-restricted $\mathrm{T}$ cell clone, DN1, using the same APCs (Figure 5A). To determine whether langerin is involved in antigen uptake or merely provides an accessory role, anti-langerin antibodies were added to cultures either before or after pulsing with antigen. For both CD1a-restricted $\mathrm{T}$ cell clones, anti-langerin antibodies blocked $\mathrm{T}$ cell proliferation only when given before the addition of antigen, not after the LC-like DCs were pulsed with antigen (Figure 5B). These data suggest that langerin is involved in the uptake of antigen for appropriate processing and presentation.

In order to determine whether freshly isolated epidermal LCs present antigen to CD1a-restricted T cells, epidermal cells were extracted from skin and used as APCs for CD1a-restricted T cell clones (Figure $5 C$ ). Epidermal cells containing LCs presented antigen to the CD1a-restricted T cell clone B2.11, whereas depletion of LCs from epidermal cell suspensions by immunomagnetic bead depletion of HLA-DR ${ }^{+}$cells completely abrogated the $\mathrm{T}$ cell response. The response of CD1a-restricted T cells to $M$. leprae in the presence of epidermal LCs was inhibited by anti-CD1a as well as anti-langerin $\mathrm{mAb}$ 's (Figure 5D), indicating that freshly isolated LCs also use langerin for CD1a-restricted antigen presentation.

Expression of langerin and CD1a on epidermal LCs in leprosy lesions. To determine whether LCs express the proteins necessary for CD1a antigen presentation in vivo, we examined the expression of CD1a and langerin in the skin lesions of leprosy patients using immunohistochemistry. Both langerin and CD1a were found on cells with dendritic morphology in the epidermis, whereas CD1b was detected in the dermis only (Figure 6A). CD1a was also expressed on cells with a dendritic morphology in the dermis, but langerin was exclusively expressed on cells in the epidermis. We next determined whether CD1a and langerin were expressed together on LCs. Langerin and CD1a were coexpressed on the dendritic-appearing cells in the epidermis (Figure 6B). In contrast, CD1b expression was limited to the dermis, and there was no colocalization with langerin. The data indicate that langerin and CD1a are present on epidermal LCs in leprosy lesions and could therefore mediate antigen presentation to $\mathrm{T}$ cells in the epidermis.

\section{Discussion}

DCs are a diverse family of APCs, each member of which has a distinct functional role. LCs represent a unique subset of DCs that act as the resident DCs in skin. Here, by comparing LCs with MD-DCs in vitro, and dermal DCs in situ, we found that LCs expressed comparatively high levels of CD1a and langerin. Furthermore, LC-like DCs were more efficient at presentation of nonpeptide antigens to $\mathrm{T}$ cells via CD1a than MD-DCs were, but less efficient at presenta- tion via CD1b. A striking finding was that langerin was required for optimal presentation of CD1a-restricted antigens by LCs. These data indicate that the expression of CD1a and langerin by LCs imparts a unique functional role for these cells in initiating immune responses to microbial pathogens.

Although it has become clear that a major function of the group 1 CD1 molecules, CD1a, CD1b, and CD1c, is to present nonpeptide antigens to $T$ cells, most of our knowledge derives from the study of CD1b- and CD1c-restricted T cells. To our knowledge, only two CD1a-restricted $\mathrm{T}$ cell clones have been described. The first CD1arestricted $T$ cell was autoreactive and derived from a patient with systemic lupus erythematosus (26). The second CD1a-restricted T cell was a CD8 ${ }^{+} \mathrm{T}$ cell clone that recognized mycobacterial antigen in the context of CD1a (22). Analysis of the CD1a antigen-presentation pathway demonstrated no requirement for endosomal localization or acidification, suggesting that CD1a traffics via recycling endosomes (27). Although previous attempts to derive $\mathrm{T}$ cell clones restricted by group 1 CD1 molecules used MD-DCs, we hypothesized that LCs, because of their high level of CD1a expression, may be more efficient at presenting antigens via CD1a. Using LC-like DCs, we derived two $M$. leprae-reactive $T$ cell clones that recognized microbial antigens in the context of CD1a presented by both in
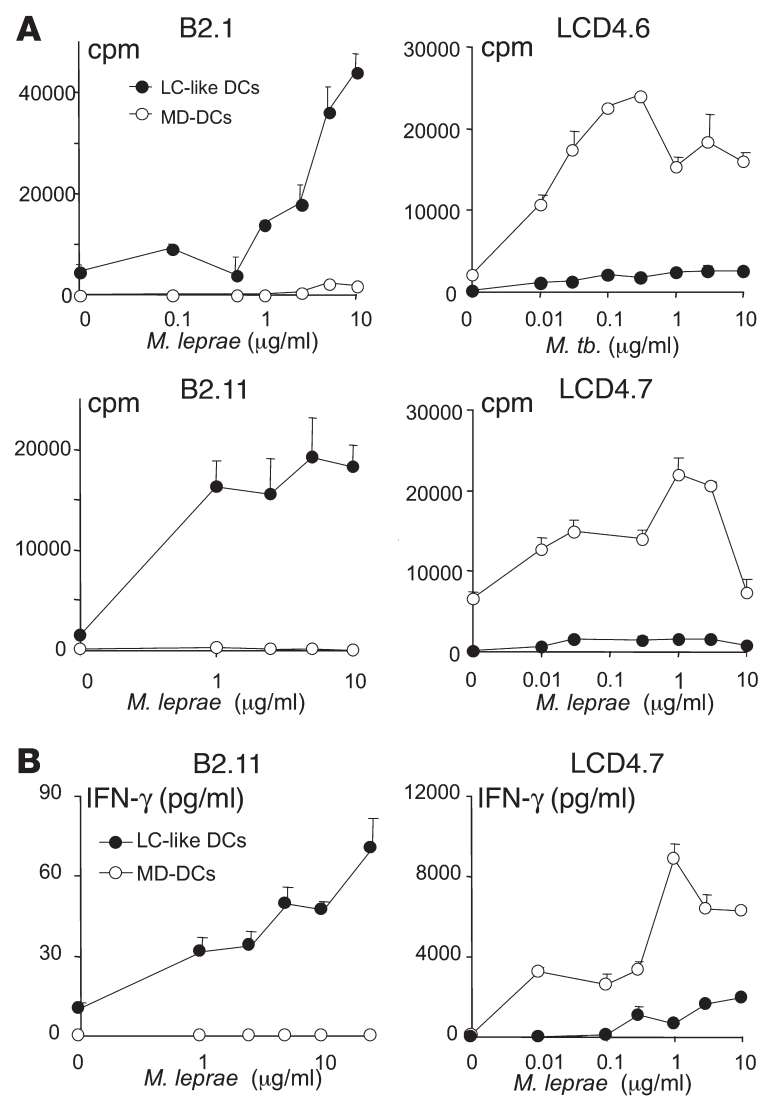

\section{Figure 4}

Comparison of antigen presentation by LC-like DCs and MD-DCs. LClike DCs (filled symbols) or MD-DCs (open symbols) were incubated with $T$ cells in the presence of the indicated amount of M. leprae or M.tuberculosis sonicates. (A) Proliferation was measured as described for Figure 2. (B) IFN- $\gamma$ production was measured as described in Methods. The values shown are the mean \pm SEM of triplicate cultures and are representative of at least three independent experiments. 


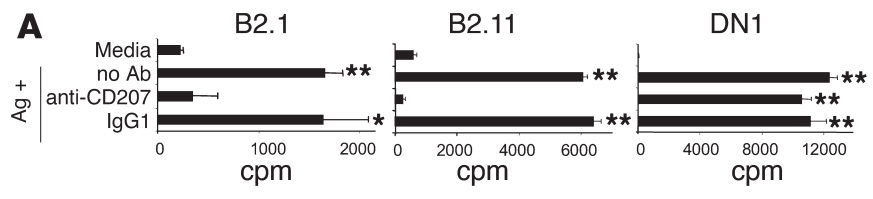

B
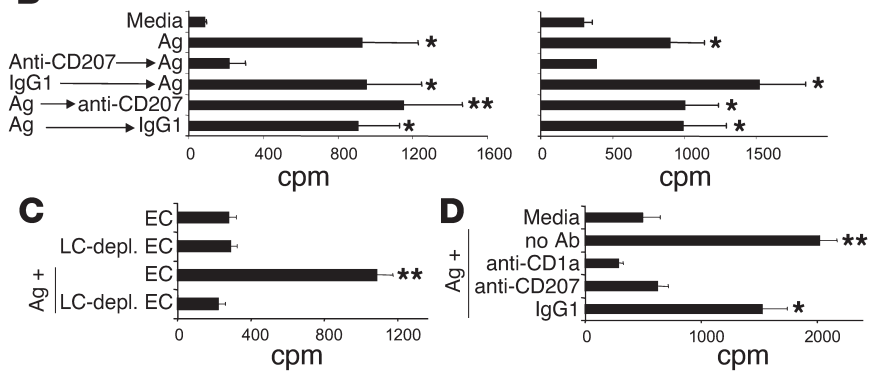

\section{Figure 5}

Influence of langerin (CD207) on antigen presentation in cord blood-derived LC-like DCs and freshly isolated epidermal LCs. (A) LClike DCs, preincubated with anti-CD207 antibodies or mouse IgG1, were cocultured with CD1a-restricted T cell clones B2.1 and B2.11 and CD1brestricted T cells (line DN1) in the presence or absence of antigen ( $M$. leprae sonicate for B2.1 and B2.11, M. tuberculosis sonicate for DN1). Proliferation was measured as described for Figure 2. (B) LC-like DCs were pulsed for 4 hours with antigen (M. leprae sonicate) or with media and cocultured with CD1a-restricted T cells. Anti-CD207 antibodies were added before (anti-CD207 $\rightarrow \mathrm{Ag}$ ) or after (Ag $\rightarrow$ anti-CD207) pulsing of the LC-like DCs with antigen. Proliferation was measured as described for Figure 2. (C) CD1a-restricted T cells (clone B2.11) were cocultured with epidermal cell suspension (EC) or EC depleted of LCs (LC-depl. EC) in the presence or absence of antigen ( $M$. leprae sonicate, $5 \mu \mathrm{g} / \mathrm{ml})$. Proliferation was measured as described for Figure 2. (D) EC, preincubated with the indicated antibodies or mouse IgG1, was cocultured with CD1arestricted T cells (clone B2.11) in the presence or absence of antigen. Proliferation was measured as described for Figure 2. The values shown are the mean \pm SEM of triplicate cultures and are representative of at least three (A) or two (B-D) independent experiments. Statistical analysis was performed as indicated in the legend to Figure 2, but comparing T cells stimulated with media versus antigen. ${ }^{\star} P<0.05 ;{ }^{\star}{ }^{\star} P<0.005$.

vitro-derived LC-like DCs and LCs isolated directly from skin. These clones recognized nonpeptide antigens, most likely glycolipid antigens, consistent with the function of group $1 \mathrm{CD} 1$ molecules. Interestingly, LC-like DCs were more efficient than MD-DCs at CD1a antigen presentation, indicating a specialized role for LCs as a skinresident $\mathrm{DC}$ subset.

A recently described feature of LCs is their specific expression of langerin (10). Langerin is a C-type lectin that can bind and mediate the uptake of sugar-containing molecules including mannose, $\mathrm{N}$-acetylglucosamine, and fucose $(28,29)$. As such, langerin is a pattern-recog-

\section{Figure 6}

LCs express both CD1a and langerin in vivo. (A) Expression of langerin (left panels), CD1a (middle panels), and CD1b (right panels) in the epidermis (upper panels) and the dermis (lower panels) of the skin lesion of a leprosy patient. The images represent sections from the lesion of one patient showing the same region of the epidermis (upper panels) and the same granuloma (lower panels). Original magnification, $\times 200$. (B) Colocalization of langerin and CD1a in the epidermis of a leprosy skin lesion. The dotted line indicates the margin between the epidermis (top) and the dermis (bottom). Original magnification, $\times 200$. nition receptor of the innate immune system, since it is germ line-encoded to recognize particular biochemical motifs. Transfection of langerin can induce the formation of Birbeck granules (11), a unique tennis racket-shaped pentilaminar granule of LCs, thought to represent a recycling/early-endosomal structure (25). We found that antibodies to langerin blocked antigen presentation by LCs to the two $T$ cell clones only if the antibodies were used to treat the LCs before addition of antigen. These data suggest that langerin imparts a specific function to LCs in facilitating antigen presentation by CD1a.

Like all CD1 proteins, CD1a is transported to the cell surface to position it for sorting events that deliver it selectively to endosomal compartments that contain microbial lipids (30). However, CD1a lacks the endosomal-localization motif found in other CD1 proteins (31) that recruits adaptor proteins to direct CD1b and CD1d to late endosomes and MHC class II compartments and CD1c to early endosomes (32). Instead, CD1a localizes to recycling endosomes, possibly through a default pathway $(27,30)$. Furthermore, CD1a has been shown to accumulate in Birbeck granules (33), where it colocalizes with langerin (11). Our data are consistent with the hypothesis that langerin serves to internalize microbial glycolipids to Birbeck granules of LCs, where the glycolipids are loaded into CD1a for presentation to $\mathrm{T}$ cells. In this manner, LCs, by the expression of CD1a, langerin, and the formation of Birbeck granules, are uniquely equipped for the uptake of specific glycolipid antigens and presentation via CD1a to $T$ cells.

We considered mycobacterial lipoarabinomannan (LAM) to be a likely candidate antigen for uptake by langerin and presentation by CD1a to T cells. Langerin binds mannose-containing molecules, and LAM has been shown to bind to and be taken up by DC-SIGN
A

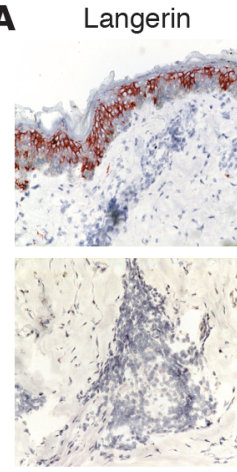

B

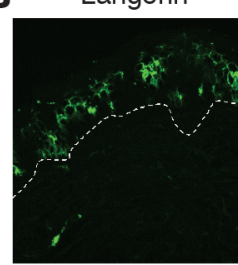

Langerin

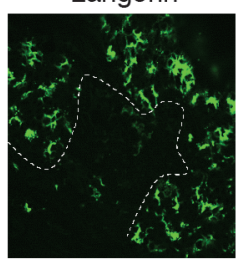

CD1a

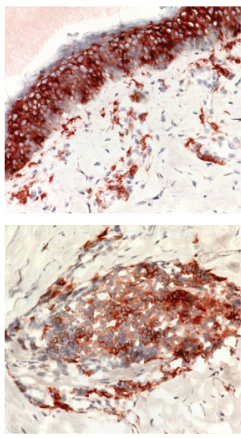

CD1a

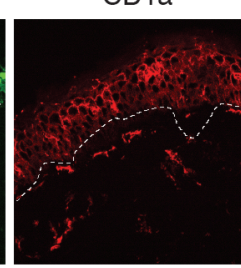

CD1b

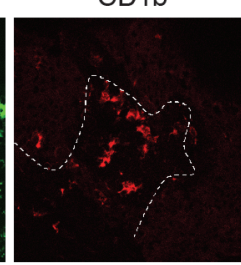

CD1b

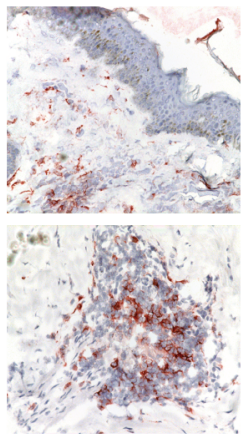

Langerin + CD1a

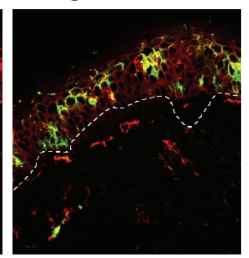

Langerin + CD1b

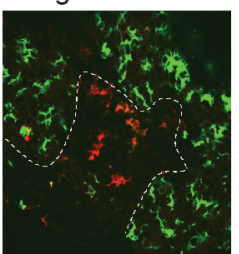


(34-36), another C-type lectin expressed by MD-DCs (37). However, the two CD1a-restricted T cells did not recognize LAM, nor any of the other previously identified lipid antigens presented by group 1 CD1 molecules, suggesting that a novel lipid antigen is presented by CD1a. Instead, the CD1a-restricted T cell clone recognized MAGP, an insoluble mycobacterial cell wall extract composed predominantly of peptidoglycan, arabinogalactan, and arabinomycolate. Overwhelming evidence indicates that CD1 presents lipid and glycolipid antigens to $\mathrm{T}$ cells $(7,9,21,38)$, and we found that the antigen was not active after protease treatment, which suggests that arabinomycolate, a glycolipid, is the likely CD1a antigen, whereas peptidoglycan and arabinogalactan, both devoid of lipid, are less likely candidates. Arabinomycolate is covalently linked to the mycobacterial cell wall and has not been isolated separately from the cell wall core peptidoglycan and galactan. Cleavage of the glycolipid from the cell wall would be required for CD1a antigen presentation; processing of glycolipids within lysosomes has been described for CD1d antigen presentation (39). We speculate that the mild acidic conditions of the early endosome act on the arabinofuranan ring structure (40), resulting in cleavage of the arabinan and release of the arabinomycolate. It is well known that the furanose form (five-member ring) of sugars is less stable than the pyranose form (six-member ring). The fatty acid chains of the glycolipids binding CD1a are likely to be shorter in length than the glycolipids presented by CD1b, as suggested by the recent determination of the structure of CD1a (41); this further suggests that a unique glycolipid is presented by CD1a. Biochemical analysis and identification of the antigen or antigens recognized by these cells will provide insight into the mechanism by which LCs use langerin and $\mathrm{CD} 1 \mathrm{a}$ to acquire antigen and present it to $\mathrm{T}$ cells.

LCs, like all DCs, are cells of the innate immune system that can influence the generation of an adaptive $\mathrm{T}$ cell response through their ability to take up proteins and to process and present them via MHC class II molecules to T cells (42). Our data indicate that LCs have a specialized role in the presentation of nonpeptide antigens to $\mathrm{T}$ cells, and that this function is mediated by the LC-specific pattern-recognition receptor langerin as well as the antigen-presenting molecule CD1a, which is highly expressed by these cells. Targeting of nonpeptide antigens to LCs is therefore a vaccine strategy that could be used for the generation of immunity to cutaneous pathogens. The location of LCs in the epidermis allows the innate immune system to respond rapidly to microbial invaders in the skin. It is notewor- thy that lesions that arise early in the course of leprosy are found in the epidermis (43), suggesting that a rapid response to epidermal infection by $M$. leprae by LCs could result in improved clearance of the organism. We conclude that subsets of DCs are highly specialized to mediate distinct functions in particular anatomic locations.

Note added in proof. Since this paper was accepted, Moody et al. (44) have described a novel mycobacterial antigen for CD1a-restricted $\mathrm{T}$ cells, termed mycobactin. In collaboration with Moody, we have examined a fraction from $M$. tuberculosis containing mycobactin and determined that the CD1a-restricted T cells we describe do not recognize mycobactin. These data further support our claims that the $\mathrm{T}$ cell clones described here recognize a unique lipid antigen. After initial submission of the manuscript, Pean-Cruz et al. (45) demonstrated a role for LCs in antigen presentation through CD1a. The present study extends the findings of Pena-Cruz and colleagues by implicating langerin in the CD1a antigen presentation pathway.

\section{Acknowledgments}

We thank Michael Brenner for helpful scientific discussions. We also thank Matthew Schibler and the Carol Moss Spivak Cell Imaging Facility in the UCLA Brain Research Institute for the use of the confocal laser microscope, and the UCLA Flow Cytometry Core Laboratory for the use of their facilities. This study was supported by grants AI22553 and AR40312 from the NIH and by the United Nations Development Programme/World Bank/WHO Special Programme for Research and Training in Tropical Diseases (to R.L. Modlin). R.E. Hunger was supported by grants from the Swiss Foundation for Medical-Biological Fellowships and the Novartis Foundation. S.A. Porcelli was supported by grants AI45889 and AI48933 from the National Institute of Allergy and Infectious Diseases (NIH), and by a Clinical Scientist Award in Translational Research from the Burroughs Wellcome Fund.

Received for publication July 29, 2003, and accepted in revised form December 16, 2003.

Address correspondence to: Robert L. Modlin, Division of Dermatology, Department of Medicine, David Geffen School of Medicine at the University of California, 10833 Le Conte Avenue, Los Angeles, California 90095, USA. Phone: (310) 825-5420; Fax: (310) 206-9878. E-mail: rmodlin@mednet.ucla.edu.
1. Klareskog, L., Tjernlund, U., Forsum, U., and Peterson, P.A. 1977. Epidermal Langerhans cells express Ia antigens. Nature. 268:248-250.

2. Rowden, G., Lewis, M.G., and Sullivan, A.K. 1977. Ia antigen expression on human epidermal Langerhans cells. Nature. 268:247-248.

3. Romani, N., et al. 1989. Presentation of exogenous protein antigens by dendritic cells to $\mathrm{T}$ cell clones. Intact protein is presented best by immature, epidermal Langerhans cells. J. Exp. Med. 169:1169-1178.

4. Cohen, P.J., and Katz, S.I. 1992. Cultured human Langerhans cells process and present intact protein antigens. J. Invest. Dermatol. 99:331-336.

5. Fithian, E., et al. 1981. Reactivity of Langerhans cells with hybridoma antibody. Proc. Natl. Acad. Sci. U. S. A. 78:2541-2544.

6. Porcelli, S., Morita, C.T., and Brenner, M.B. 1992. $\mathrm{CD} 1 \mathrm{~b}$ restricts the response of human CD4-8- T lymphocytes to a microbial antigen. Nature. 360:593-597.

7. Sieling, P.A., et al. 1995. CD1-restricted T cell recognition of microbial lipoglycans. Science. 269:227-230.

8. Moody, D.B., et al. 1997. Structural requirements for glycolipid antigen recognition by CD1b-restricted T cells. Science. 278:283-286.

9. Moody, D.B., et al. 2000. CD1c-mediated T-cell recognition of isoprenoid glycolipids in Mycobacterium tuberculosis infection. Nature. 404:884-888.

10. Valladeau, J., et al. 1999. The monoclonal antibody DCGM4 recognizes Langerin, a protein specific of Langerhans cells, and is rapidly internalized from the cell surface. Eur. J. Immunol. 29:2695-2704.

11. Valladeau, J., et al. 2000. Langerin, a novel C-type lectin specific to Langerhans cells, is an endocytic receptor that induces the formation of Birbeck granules. Immunity. 12:71-81.

12. Ridley, D.S., and Jopling, W.H. 1966. Classification of leprosy according to immunity. A five-group system. Int. J. Lepr. 34:255-273.

13. Beckman, E.M., et al. 1996. CD1c restricts responses of mycobacteria-specific T cells. Evidence for antigen presentation by a second member of the human CD1 family. J. Immunol. 157:2795-2803.

14. Fairhurst, R.M., Wang, C.X., Sieling, P.A., Modlin, R.L., and Braun, J. 1998. CD1 presents antigens from a gram-negative bacterium, Haemophilus influenzae type B. Infect. Immun. 66:3523-3526.

15. Reinherz, E.L., Kung, P.C., Goldstein, G., Levey, R.H., and Schlossman, S.F. 1980. Discrete stages of human intrathymic differentiation: analysis of normal thymocytes and leukemic lymphoblasts of T-cell lineage. Proc. Natl. Acad. Sci. U. S. A. 77:1588-1592.

16. Behar, S.M., Porcelli, S.A., Beckman, E.M., and Brenner, M.B. 1995. A pathway of costimulation that prevents anergy in CD28- T cells: B7-independent costimulation of CD1-restricted T cells. J. Exp. Med. 182:2007-2018.

17. Melian, A., Geng, Y.J., Sukhova, G.K., Libby, P., and Porcelli, S.A. 1999. CD1 expression in human atherosclerosis. A potential mechanism for $\mathrm{T}$ cell activation by foam cells. Am. J. Pathol. 155:775-786.

18. Kasinrerk, W., Baumruker, T., Majdic, O., Knapp, W., and Stockinger, H. 1993. CD1 molecule expression on human monocytes induced by granulocytemacrophage colony-stimulating factor. J. Immunol. 150:579-584.

19. Blauvelt, A., Glushakova, S., and Margolis, L.B. 2000. HIV-infected human Langerhans cells transmit infection to human lymphoid tissue ex vivo. AIDS. 14:647-651.

20. Mehra, V., et al. 1992. A major T cell antigen of Mycobacterium leprae is a $10-\mathrm{kD}$ heat-shock cognate 
protein. J. Exp. Med. 175:275-284.

21. Beckman, E.M., et al. 1994. Recognition of a lipid antigen by CD1-restricted $\alpha \beta+\mathrm{T}$ cells. Nature. 372:691-694.

22. Rosat, J.P., et al. 1999. CD1-restricted microbial lipid antigen-specific recognition found in the CD $8+\alpha \beta$ T cell pool. J. Immunol. 162:366-371.

23. Ochoa, M.T., et al. 2001. T-cell release of granulysin contributes to host defense in leprosy. Nat. Med. 7:174-179.

24. Tanaka, Y., et al. 1994. Nonpeptide ligands for human gamma delta T cells. Proc. Natl. Acad. Sci. U. S. A. 91:8175-8179.

25. McDermott, R., et al. 2002. Birbeck granules are subdomains of endosomal recycling compartment in human epidermal Langerhans cells, which form where Langerin accumulates. Mol. Biol. Cell. 13:317-335.

26. Porcelli, S., et al. 1989. Recognition of cluster of differentiation 1 antigens by human CD4-CD8-cytolytic T lymphocytes. Nature. 341:447-450.

27. Sugita, M., et al. 1999. Separate pathways for antigen presentation by CD1 molecules. Immunity. 11:743-752.

28. Stambach, N.S., and Taylor, M.E. 2003. Characterization of carbohydrate recognition by langerin, a Ctype lectin of Langerhans cells. Glycobiology. 13:401-410.

29. Plzak, J., et al. 2002. Analysis of binding of mannosides in relation to Langerin (CD207) in Langerhans cells of normal and transformed epithelia. His- tochem. J. 34:247-253.

30. Moody, D.B., and Porcelli, S.A. 2003. Intracellular pathways of CD1 antigen presentation. Nat. Rev. Immunol. 3:11-22.

31. Jackman, R.M., et al. 1998. The tyrosine-containing cytoplasmic tail of CD1b is essential for its efficient presentation of bacterial lipid antigens. Immunity. 8:341-351.

32. Sugita, M., et al. 2002. Failure of trafficking and antigen presentation by CD1 in AP-3-deficient cells. Immunity. 16:697-706.

33. Hanau, D., et al. 1987. Human epidermal Langerhans cells cointernalize by receptor-mediated endocytosis "nonclassical" major histocompatibility complex class I molecules (T6 antigens) and class II molecules (HLA-DR antigens). Proc. Natl. Acad. Sci. U. S. A. 84:2901-2905.

34. Geijtenbeek, T.B., et al. 2003. Mycobacteria target DC-SIGN to suppress dendritic cell function. J. Exp. Med. 197:7-17.

35. Tailleux, L., et al. 2003. DC-SIGN is the major Mycobacterium tuberculosis receptor on human dendritic cells. J. Exp. Med. 197:121-127.

36. Maeda, N., et al. 2003. The cell surface receptor DCSIGN discriminates between Mycobacterium species through selective recognition of the mannose caps on lipoarabinomannan. J. Biol. Chem. 278:5513-5516.

37. Geijtenbeek, T.B., et al. 2000. Identification of DCSIGN, a novel dendritic cell-specific ICAM-3 receptor that supports primary immune responses.
Cell. 100:575-585.

38. Kawano, T., et al. 1997. CD1d-restricted and TCRmediated activation of $v \alpha 14$ NKT cells by glycosyl ceramides. Science. 278:1626-1629.

39. Prigozy, T.I., et al. 2001. Glycolipid antigen processing for presentation by CD1d molecules. Science. 291:664-667.

40. Daffe, M., Brennan, P.J., and McNeil, M. 1990. Predominant structural features of the cell wall arabinogalactan of Mycobacterium tuberculosis as revealed through characterization of oligoglycosyl alditol fragments by gas chromatography/mass spectrometry and by $1 \mathrm{H}$ and $13 \mathrm{C}$ NMR analyses. J. Biol. Chem. 265:6734-6743.

41. Zajonc, D.M., Elsliger, M.A., Teyton, L., and Wilson, I.A. 2003. Crystal structure of CD1a in complex with a sulfatide self antigen at a resolution of 2.15 A. Nat. Immunol. 4:808-815.

42. Stingl, G., Katz, S.I., Clement, L., Green, I., and Shevach, E.M. 1978. Immunologic functions of Ia-bearing epidermal Langerhans cells. J. Immunol. 121:2005-2013.

43. Ridley, D.S. 1972. The pathogenesis of the early lesions in leprosy. J. Pathol. 111:191-206.

44. Moody, D.B., et al. 2004. T cell activation by lipopeptide antigens. Science. 303:527-531.

45. Pena-Cruz, V., Ito, S., Dascher, C.C., Brenner, M.B., and Sugita, M. 2003. Epidermal Langerhans cells efficiently mediate CD1a-dependent presentation of microbial lipid antigens to T cells. J. Invest. Dermatol. 121:517-521 